\title{
悬 \\ Predação de animais domésticos em pequenas propriedades rurais do entorno de áreas protegidas da Mata Atlântica do Estado de Pernambuco, Brasil
}

\author{
[Livestock predation on small rural properties near protected areas of the Atlantic Forest of \\ Pernambuco State, Brazil]
}

\section{"Artigo Científico/Scientific Article"}

\author{
Diogo Lima Franco ${ }^{1,2}$, Rafael Lima Oliveira ${ }^{2,3}$, Ricardo César Souza Carneiro da Cunha ${ }^{2}$, \\ Vanessa Oliveira Ribeiro $^{2,4}$, Maria Fernanda Vianna Marvulo ${ }^{4,5}$, Jean Carlos Ramos Silva ${ }^{2,4}$
}

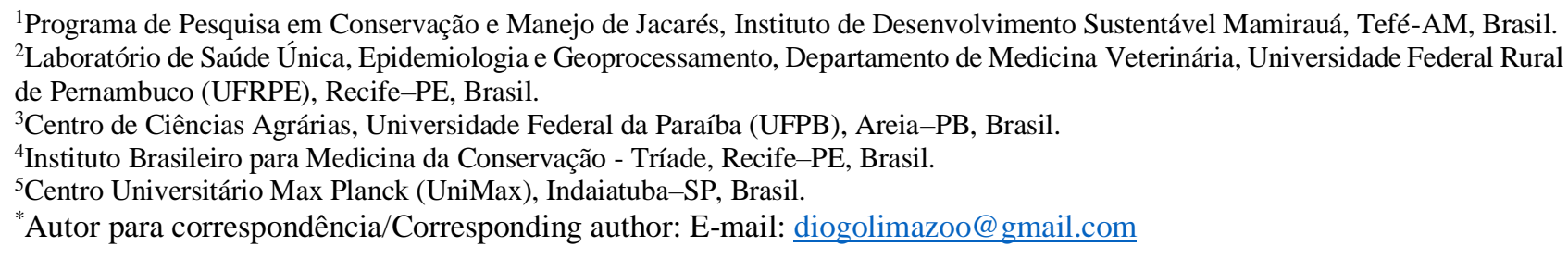

\section{Resumo}

O objetivo desse estudo foi caracterizar a ocorrência de predação a rebanhos domésticos em pequenas propriedades rurais do entorno de áreas protegidas da Mata Atlântica do Estado de Pernambuco, Brasil. Ao todo, foram visitadas 11 pequenas propriedades próximas à Estação Ecológica de Tapacurá (n=6), em São Lourenço da Mata, e à área protegida da $10^{\mathrm{a}}$ Brigada de Infantaria Motorizada $(\mathrm{n}=5)$, em Recife. Nas propriedades, foram aplicados questionários aos proprietários, no intuito de obter dados sobre os ataques ocorridos aos animais domésticos por animais silvestres, assim como, obter informações sobre as características ambientais da propriedade, espécies criadas, tamanho do rebanho, sistemas de criação e manejo adotado. Em 54,5\% (6/11) das propriedades foram relatados casos de predação. As galinhas foram predadas em $60 \%$ (9/15) dos casos e os cachorros-do-mato (Cerdocyon thous) foram os principais predadores de animais domésticos, responsáveis por $40 \%$ (4/15) dos ataques. Foi observada correlação negativa entre a ocorrência de predação e a presença de cercas-vivas na propriedade e a distância da propriedade em relação à área protegida, e positiva entre ocorrência de predação e fontes naturais de água na propriedade.

Palavras-chave: conflitos homem-animais silvestres; aves domésticas; cachorro-do-mato; teiú; área protegida.

\begin{abstract}
The objective of this study was to characterize the occurrence of livestock predation in small rural properties surrounding protected areas of the Atlantic Forest in the State of Pernambuco, Brazil. In total, 11 small properties were visited near the Ecologic Station of Tapacurá $(n=6)$, in São Lourenço da Mata, and the protected area of the $10^{\text {th }}$ Motorized Infantry Brigade $(n=5)$, in Recife. The owners of the properties were surveyed with the intention of collecting data regarding the attacks against small domestic animals by wild animals, as well as to obtain information on the environmental characteristics of the property, species bred, herd size, and breeding and management systems adopted. In $54.5 \%(6 / 11)$ of the properties, cases of predation were reported. Chickens were the prey in $60 \%$ (9/15) of the cases and crab-eating foxes (Cerdocyon thous) were the main predators of livestock, responsible for $40 \%$ (4/15) of the attacks. A negative correlation was observed between the predation and the presence of hedgerows in the property and the distance between the property in relation to the protected areas. A positive correlation was observed between the predation and the natural water supply in the property.
\end{abstract}

Keywords: human-wildlife conflicts; poultry; crab-eating fox; tegu lizard; protected areas. 


\section{Introdução}

Diversos fatores ecológicos e antrópicos influenciam no declínio global das espécies carnívoras selvagens, diminuindo suas áreas de ocorrência natural (Patterson et al., 2004; Gese, 2006; Reis et al., 2006). A sua necessidade de grandes áreas de vida (Muñoz-Garcia e Williams, 2005) e dependência de outros animais como fonte de alimento, expõe os predadores a conflito direto com os seres humanos, principalmente em áreas perturbadas e onde são forçados a conviver com animais domésticos (Pitman et al., 2002; Distefano, 2005). Essa proximidade pode ocasionar a predação dos rebanhos domésticos, havendo inclusive casos em que esses representam parte predominante da dieta dos predadores (Carreira e Petrucci-Fonseca, 2000; Azevedo, 2006), acarretando perdas econômicas e desequilíbrio nas relações ecológicas locais (Palmeira e Barrella, 2007). Em estudo realizado por Mishra (1997) em três vilarejos na Índia, 18\% (189/1050) dos rebanhos foi perdido para animais selvagens em 18 meses, representando metade da renda per capita anual das famílias locais.

Embora os conflitos entre homens e predadores sejam antigos, as informações são insuficientes para determinar os padrões de ocorrência de ataques (Graham et al., 2004). Enquanto isso, em todo o mundo, esses conflitos acarretam a morte de um grande número de carnívoros selvagens em retaliação aos danos causados à pecuária (Ogada et al., 2003; Distefano, 2005; IUCN, 2018), que poderiam ser minimizados com a adoção de práticas simples de manejo, como confinamento noturno e isolamento de acesso do rebanho à áreas naturais (Marchini et al., 2011). Os aspectos zootécnicos, como grau de confinamento, características do rebanho, intensidade do manejo e do acompanhamento e controle dos rebanhos, podem ter influência significativa nas ocorrências de predação (Ogada et al., 2003; Kolowski e Holekamp, 2006; Michalski et al., 2006) e a análise destes fatores possibilita a identificação de pontoschave para a prevenção dos ataques (Tortato et al., 2015).

Em diversos países vêm sendo adotadas práticas preventivas há muitos anos, com relativa eficácia na redução de ataques de predadores, com baixo custo e sem a necessidade de utilização de métodos letais de controle ou translocação (Pitman et al., 2002; Ogada et al., 2003; Distefano, 2005; Gese, 2006). A eficácia das medidas de controle depende da adequação destas a cada local, tendo em vista a grande diversidade de animais responsáveis por ataques (Carreira e PetrucciFonseca, 2000; Patterson et al., 2004; Vidolin et al., 2004; Distefano, 2005; Silva-Rodríguez et al., 2009) e das características dos rebanhos criados e manejo utilizado (Kolowski e Holekamp, 2006; Michalski et al., 2006; Gese, 2006).

Os estudos realizados em relação a ataques às criações de animais domésticos no Brasil são em sua maioria realizados em grandes propriedades das regiões Centro-Oeste, Sudeste e Sul (Vidolin et al., 2004; Hoogesteijn e Hoogesteijn, 2011; Tortato et al., 2015). Mesmo considerando que perdas de animais domésticos ocasionam aos pequenos produtores maiores danos econômicos e riscos à segurança alimentar (Mishra, 1997; Distefano, 2005; Silva-Rodríguez et al., 2009), estudos sobre ocorrência e impacto da predação de rebanhos domésticos na pecuária familiar são escassos no Brasil (Palmeira e Barrella, 2007). A agricultura familiar representa $84,4 \%$ do total de propriedades agropecuárias do país, com cerca de 50\% (2.187.295 de propriedades) destas na região Nordeste (BRASIL, 2006), indicando a necessidade de pesquisas específicas sobre a predação em pequenas propriedades de produção familiar, assim como a compreensão destes conflitos em outras regiões do país.

Desse modo, este estudo objetivou realizar um levantamento e caracterização da ocorrência de predação a rebanhos domésticos em pequenas propriedades rurais localizadas no entorno de áreas protegidas da Mata Atlântica do Estado de Pernambuco, Nordeste, Brasil.

\section{Material e Métodos}

Foram visitadas 11 pequenas propriedades rurais (máximo de 10 hectares) próximas à duas áreas protegidas (AP) de Mata Atlântica localizadas na Região Metropolitana do Recife, Estado de Pernambuco, sendo seis propriedades próximas à Estação Ecológica do Tapacurá e cinco próximas à10 $0^{\mathrm{a}}$ Brigada de Infantaria Motorizada. A Estação Ecológica do Tapacurá - ESEC Tapacurá (Latitude: $8^{\circ} 02^{\prime} \mathrm{S}$, Longitude: $35^{\circ} 11^{\prime} \mathrm{O}$ ) localizada no município de São Lourenço da Mata, ocupa uma área de 776 hectares de Mata Atlântica circundada por florestas secundárias, campos e canaviais. $\mathrm{O}$ clima é do tipo "Aw" com precipitação média anual de $1.300 \mathrm{~mm}$ e cinco meses secos (setembro a janeiro) com menos de $100 \mathrm{~mm}$ de precipitação. As propriedades visitadas são parte de um 
assentamento rural circunvizinho aos limites da ESEC.

A área protegida (AP) da $10^{\mathrm{a}}$ Brigada de Infantaria Motorizada - $10^{\mathrm{a}}$ BDAINFMTZ (Latitude $08^{\circ} 04^{\prime} \mathrm{S}$, Longitude: $34^{\circ} 57^{\prime} \mathrm{O}$ ) possui cerca de 100 hectares de Mata Atlântica formada por florestas secundárias, inseridas na área urbana do município de Recife, as margens da BR-232. O clima é do tipo "Am", com precipitação média anual de $1.757 \mathrm{~mm}$ e três meses com menos de 60 $\mathrm{mm}$ de precipitação (outubro a dezembro). As propriedades visitadas encontraram-se no interior da AP.

No total, foram realizadas expedições às áreas de estudo, com duração média de oito dias por mês, entre julho de 2010 e junho de 2011. Em todas as áreas de estudo foram realizados reconhecimentos prévios e visitas monitoradas, para verificação de ocorrência de vestígios, por meio da identificação de pegadas, fezes, marcas de garras em troncos, odores, sinais de alimentação e visualização, de modo a confirmar a ocorrência de possíveis espécies de predadores na região (Pitman et al., 2002; Reis et al., 2006).

Durante as visitas às propriedades foram aplicados questionários semi-estruturados aos proprietários, visando obter dados sobre possíveis ataques ocorridos na propriedade e identificar fatores de risco para predação (Pitman et al., 2002; Graham et al., 2004; Distefano, 2005; Azevedo, 2006; Soto-Shoender e Giuliano, 2011): área da AP próxima; distância da AP; área da propriedade; presença de fontes naturais de água; ausência de cercas (arame e cerca-viva); manejo de cadáveres inadequado (animais mortos deixados ao ar livre); manejo extensivo, grandes plantéis e ausência de cães. As análises de estatística descritiva e teste de correlação foram realizadas no software BioEstat 5.3 .

\section{Resultados}

Das 11 propriedades visitadas, em seis $(54,5 \%)$ foram relatados casos de predação de animais domésticos (Tabela 1), quatro destas próximas à área da $10^{\mathrm{a}}$ Brigada de Infantaria Motorizada e duas próximas à ESEC Tapacurá. Os ataques foram citados como frequentes, porém as datas de ocorrência e o total de animais atacados não foram informados, pois apenas $18 \%$ dos 11 entrevistados possuíam algum tipo de controle zootécnico, como livro de registro e acompanhamento diário do plantel.
De um rebanho total de 313 animais nas 11 propriedades, $84 \%$ eram de pequeno porte. Os animais mais comumente criados foram galinhas (Gallus gallus domesticus) (plantel médio de 23 animais por propriedade), presentes em $100 \%$ das propriedades; bovinos (Bos taurus) (média de oito por propriedade) em $36 \%$ das propriedades; caprinos (Capra hircus) (média de três animais por propriedade) em $27 \%$ das propriedades e patos (Cairina moschata) (média de dois animais por propriedade) em $18 \%$ das propriedades. Foram relatados ataques sobre galinhas em $83 \%(5 / 6)$ das propriedades com ocorrências de predação; sobre ovos em 33\% (2/6) e patos em 17\% (1/6).

Considerando os ataques com avistamento de predador nos últimos 12 meses (15 relatos), as galinhas foram predadas em 60\% (9/15) dos casos, seguidas por ovos $(26 \%$; $4 / 15)$ e patos $(14 \% ; 2 / 15)$. Um total de 17 animais domésticos foi abatido nos últimos incidentes de predação, excluindo-se as perdas de ovos, que não foram contabilizadas em nenhuma propriedade (Tabela 2).

$\mathrm{O}$ número de perdas por propriedade em um único ataque variou de um a cinco indivíduos, chegando a representar 37,5\% (3/8) do rebanho de uma das propriedades. Cerca de 65\% (11/17) dos animais abatidos foram crias e jovens, as únicas categorias que tiveram abatidos mais de um animal por ataque. Todas as espécies de predadores relatadas nas propriedades em Pernambuco foram confirmadas por meio de rastreamento, avistamentos e publicações científicas (Pitman et al., 2002; Reis et al., 2006; IUCN, 2018).

Os cachorros-do-mato (Cerdocyon thous) foram os principais predadores de animais domésticos nas propriedades visitadas, responsáveis por $40 \%(6 / 15)$ dos últimos ataques com avistamento do predador. Irara (Eira barbara), guaxinim (Procyon cancrivorus), gambá (Didelphis spp.) e teiú (Salvator merianae) também foram predadores citados. Embora a espécie de serpente constritora (família Boidae) responsável pelo ataque não tenha sido identificada pelo entrevistado, ocorrem na área de estudo a jiboia (Boa constrictor) e a salamanta (Epicrates cenchria). O jacaré (família Alligatoridae) também não foi identificado, porém ocorrem na área de estudo o jacaré-de-papo-amarelo (Caiman latirostris) e o jacaré-paguá (Paleosuchus palpebrosus). O saimiri (Saimiri sciureus) e o cãodoméstico (Canis lupus familiaris), espécies exóticas introduzidas, também foram avistados 
predando animais domésticos, em 14\% (2/15) e 7\% (1/15) dos ataques, respectivamente (Tabela 2).

Fatores de risco à predação (FRP) foram observados em $91 \%$ das 11 propriedades visitadas. As correlações entre ocorrência de predação e presença de cercas-vivas $\left(\mathrm{r}^{2}=-0,6708, \mathrm{p}<0,05\right)$, distância da propriedade em relação à área protegida e $\left(\mathrm{r}^{2}=-0,5784, \mathrm{p}<0,1\right)$ e presença de fontes naturais de água dentro da propriedade $\left(\mathrm{r}^{2}=\right.$ $0,559, \mathrm{p}<0,1)$ mostraram significância. Todos os ataques ocorreram em propriedades a até $1,5 \mathrm{~km}$ das áreas protegidas, 67\% (4/6) deles nas propriedades no interior de AP (Tabela 3).

Em todas as propriedades onde se encontrou uma fonte de água natural ocorreram predações. Cerca de 55\% (6/11) das propriedades visitadas tinham até 1 hectare de área, porém $67 \%$ (4/6) das que relataram ataques tinham entre 5 e 10 ha. $\mathrm{O}$ tamanho dos rebanhos atacados variou de oito a 60 aves, sendo mais comuns (67\% das ocorrências) os ataques sobre plantéis com mais de 20 animais (Tabela 1).

A principal forma de manejo utilizada nas áreas com ocorrências de predação foi o manejo extensivo $(50 \% ; 3 / 6)$, com o rebanho tendo acesso a toda a área da propriedade e arredores durante todo o dia. O manejo intensivo foi utilizado em $33 \%$ (2/6) das propriedades com predação e a contenção noturna em $17 \%$ (1/6). As propriedades sem ocorrência de predação utilizavam em sua maioria $(80 \% ; 4 / 5)$ o sistema de confinamento noturno.

Das propriedades atacadas, $83 \% \quad(5 / 6)$ possuíam cerca de arame farpado constituída em média por cinco fios, $20 \mathrm{~cm}$ equidistantes. As propriedades cercadas por cercas-vivas de cactáceas $(27 \%$; 3/11) não sofreram nenhuma perda. A troca da cerca de arame por cerca telada mitigou por completo os ataques em uma das propriedades.

Os animais mortos foram enterrados em 83\% (5/6) das propriedades com relatos de predação. Cães domésticos estavam presentes em $83 \%$ (5/6) dos locais atacados, em números de até cinco animais. Quanto à época com maior concentração de ocorrências, três proprietários (50\%), dois em São Lourenço da Mata e um em Recife, declararam ser na época de chuva quando a maioria dos ataques acontece, enquanto para os outros três proprietários em Recife informaram que os ataques aconteceram sem sazonalidade.

Tabela 1. Características ambientais e produtivas das 11 pequenas propriedades rurais no entorno de áreas protegidas na Mata Atlântica de Pernambuco.

\begin{tabular}{|c|c|c|c|c|c|c|c|c|c|c|c|}
\hline \multirow[b]{2}{*}{ Variáveis } & \multicolumn{11}{|c|}{ Propriedades } \\
\hline & \multicolumn{6}{|c|}{ ESEC Tapacurá $^{1}$} & \multicolumn{5}{|c|}{$10^{\mathrm{a}} \mathrm{BDAINFMTZ}^{2}$} \\
\hline Predação & $\operatorname{sim}$ & $\operatorname{sim}$ & não & não & não & não & $\operatorname{sim}$ & $\operatorname{sim}$ & $\operatorname{sim}$ & não & $\operatorname{sim}$ \\
\hline Galinha $\left(\mathrm{n}^{3}\right)$ & 20 & 8 & 6 & 4 & 10 & 5 & 18 & 50 & 25 & 50 & 60 \\
\hline Bovino (n) & - & 3 & 3 & - & - & - & 8 & 18 & - & - & - \\
\hline Equino (n) & - & 3 & - & - & - & - & - & - & - & - & - \\
\hline Suíno (n) & - & - & - & - & 5 & - & - & - & - & - & - \\
\hline Caprino (n) & - & 3 & - & 4 & 2 & - & - & - & - & - & - \\
\hline Pato (n) & - & - & - & 3 & - & - & - & 1 & - & - & - \\
\hline Ganso (n) & - & - & - & - & - & - & - & - & 2 & - & - \\
\hline Peru (n) & - & - & - & 2 & - & - & - & - & - & - & - \\
\hline Área da $\mathrm{AP}^{4}$ (ha) & 776 & 776 & 776 & 776 & 776 & 776 & 100 & 100 & 100 & 100 & 100 \\
\hline Distância da AP (m) & 850 & 850 & 1100 & 1100 & 1100 & 1100 & 0 & 0 & 0 & 0 & 0 \\
\hline Área da propriedade & 10 & 10 & 5 & 1 & 1 & 1 & 5 & 5 & 1 & 1 & 1 \\
\hline $\begin{array}{l}\text { Tamanho do plantel } \\
\text { (animais de pequeno } \\
\text { porte) }\end{array}$ & 20 & 8 & 6 & 7 & 10 & 5 & 18 & 51 & 25 & 50 & 60 \\
\hline Cerca de arame & $\operatorname{sim}$ & $\operatorname{sim}$ & não & $\operatorname{sim}$ & $\operatorname{sim}$ & $\operatorname{sim}$ & $\operatorname{sim}$ & $\operatorname{sim}$ & não & não & $\operatorname{sim}$ \\
\hline Cerca-viva & não & não & não & $\operatorname{sim}$ & $\operatorname{sim}$ & $\operatorname{sim}$ & não & não & não & não & não \\
\hline Manejo extensivo & $\operatorname{sim}$ & $\operatorname{sim}$ & $\operatorname{sim}$ & $\operatorname{sim}$ & não & $\operatorname{sim}$ & não & $\operatorname{sim}$ & não & não & não \\
\hline Cães (n) & 2 & 4 & 2 & 2 & 3 & - & 1 & 2 & 5 & 3 & 2 \\
\hline Fonte natural água & $\operatorname{sim}$ & $\operatorname{sim}$ & não & não & não & não & $\operatorname{sim}$ & não & não & não & não \\
\hline Cadáveres enterrados & não & $\operatorname{sim}$ & não & $\operatorname{sim}$ & $\operatorname{sim}$ & não & $\operatorname{sim}$ & $\operatorname{sim}$ & $\operatorname{sim}$ & $\operatorname{sim}$ & $\operatorname{sim}$ \\
\hline
\end{tabular}

${ }^{1}$ Estação Ecológica de Tapacurá; ${ }^{2} 10^{\mathrm{a}}$ Brigada de Infantaria Motorizada; ${ }^{3} \mathrm{n}=$ número de animais, ${ }^{4}$ área protegida 
Tabela 2. Predadores e animais atacados em 11 pequenas propriedades rurais no entorno de áreas protegidas na Mata Atlântica de Pernambuco.

\begin{tabular}{lccclc}
\hline \multirow{2}{*}{ Predador } & \multicolumn{3}{l}{$\begin{array}{l}\text { Ataques com avistamento } \\
\left(\mathrm{n}^{1}\right)\end{array}$} & \multicolumn{3}{l}{ Animais abatidos } \\
\cline { 2 - 6 } & Galinha & Pato & Ovo & Espécie & $\mathrm{n}$ \\
\hline Cachorro-do-mato (Cerdocyon thous) & 4 & - & 2 & galinha (cria/adulto) & 6 \\
Cão doméstico (Canis lupus familiaris) & - & - & 1 & ovo $^{2}$ & - \\
Irara (Eira barbara) & 1 & - & - & galinha (adulto) & 1 \\
Guaxinim (Procyon cancrivorus) & - & 1 & - & pato (adulto) & 1 \\
Gambá (Didelphis spp) & 1 & - & - & galinha (cria) & 1 \\
Saimiri (Saimiri sciureus) & 1 & - & 1 & galinha (cria) & 1 \\
Teiú (Salvator merianae) & 1 & - & - & galinha (cria) & 5 \\
Serpente constritora (família Boidae) & 1 & - & - & galinha (adulto) & 1 \\
Jacaré (família Alligatoridae) & - & 1 & - & pato (adulto) & 1 \\
Total & 9 & 2 & 4 & - & \\
\hline
\end{tabular}

${ }^{1} \mathrm{n}=$ número. ${ }^{2}$ perdas de ovos não foram contabilizadas

Tabela 3. Correlação entre os fatores de risco e a ocorrência de predação em 11 pequenas propriedades rurais no entorno de áreas protegidas na Mata Atlântica de Pernambuco

\begin{tabular}{lcc}
\hline Fatores de risco & $\mathrm{r}^{\mathbf{2}}$ & $\mathrm{p}$ \\
\hline Área da AP & $-0,4667$ & 0,1478 \\
Distância da AP & $-0,5784$ & 0,0622 \\
Área da propriedade & 0,5154 & 0,1046 \\
Tamanho do plantel & 0,3850 & 0,2422 \\
Cerca de arame & 0,2609 & 0,4384 \\
Cerca-viva & $-0,6708$ & 0,0238 \\
Manejo extensivo & $-0,1000$ & 0,7699 \\
Cães & 0,2557 & 0,4480 \\
Fonte natural de água & 0,5590 & 0,0737 \\
Cadáveres enterrados & 0,2609 & 0,4384 \\
\hline
\end{tabular}

${ }^{1}$ Área protegida

\section{Discussão}

A maior ocorrência de predação sobre aves e outros animais de pequeno porte também foi observada em trabalhos no Chile (Silva-Rodríguez et al., 2009), Brasil (Mendonça et al., 2011) e México (Amador-Alcalá et al., 2013). Resultado diferente do observado por Palmeira e Barrela (2007) em comunidades quilombolas em uma área de 9.000 ha de Mata Atlântica no Estado de São Paulo, onde onças-pintadas (Panthera onca) e onças-pardas (Puma concolor) foram responsáveis por todos os ataques, $69,5 \%$ (48/69) destes sobre suínos e equinos, mesmo quando o plantel de aves respondia por $65 \%$ (549/840) do rebanho total.

Nas propriedades visitadas em Pernambuco, animais domésticos de porte maior não foram predados possivelmente devido à redução de áreas naturais preservadas, necessárias paras a manutenção de populações de grandes carnívoros (Carreira e Petrucci-Fonseca, 2000; Vidolin et al., 2004; Muñoz-Garcia e Williams, 2005; Mendonça et al., 2011).
O maior número de predadores silvestres de porte menor (Pitman et al., 2002; Reis et al., 2006; Marchini et al., 2011; IUCN, 2018) e a grande representatividade de plantéis de aves em sistema de manejo extensivo rústico, podem explicar a ocorrência de ataques apenas sobre aves (Stahl et al., 2002; Marchini et al., 2011). O cachorro-domato apareceu como um dos principais predadores causadores de conflitos, assim como encontrado por Mendonça et al. (2011) no Estado da Paraíba, onde também foram citados guaxinim, teiú e serpentes.

Considerando o número de animais abatidos por ataque em Pernambuco, o teiú causou as maiores perdas em um único evento (Tabela 2). Não foram encontrados relatos científicos de ataques a animais domésticos por jacarés, gambás, primatas e cães domésticos na região Nordeste do país. Todos os predadores silvestres citados são generalistas, assim como encontrado por Graham et al. (2004). Estes sobrevivem em uma grande variedade de ambientes e com dietas flexíveis e oportunistas (Williams, 2005; Reis et al., 2006; 
Muñoz-Garcia e IUCN, 2018), o que permite sua adaptação a áreas perturbadas e a presença humana.

A ausência de ataques observada em propriedades com cercas-vivas corrobora outras pesquisas onde se verificou que as cercas mais eficientes foram aquelas que dificultaram a passagem e reduziram o contato visual entre predador e presa (Ogada et al., 2003; Distefano, 2005). O maior número de predações em propriedades mais próximas de áreas naturais também foi encontrado em trabalhos na Guatemala (Soto-Shoender e Giuliano, 2011) e Pantanal (Azevedo e Murray, 2007; Tortato et al., 2015) e pode ser explicado pela maior proximidade entre os predadores e os rebanhos domésticos (Pitman et al., 2002; Hoogesteijn e Hoogesteijn, 2011).

Predadores pequenos e mais generalistas tendem a ter áreas de vida menores (Graham et al., 2004; Muñoz-Garcia e Williams, 2005). Dessa forma quanto mais próximas de áreas naturais estiverem as propriedades, maior a probabilidade de que as áreas de uso dos rebanhos e dos animais silvestres se sobreponham, aumentando o risco de predação (Azevedo e Murray 2007). A correlação entre existência de fontes de água nas propriedades e ataques aos rebanhos também foi encontrada por Soto-Shoender e Giuliano (2011). Corpos de água são utilizados por predadores para matar a sede ou em busca de presas (Pitman et al., 2002; Hoogesteijn e Hoogesteijn, 2011), o que pode explicar essa correlação.

O tamanho da área protegida, a área da propriedade e tamanho do rebanho mostraram níveis intermediários de significância $(\mathrm{p}<0,3)$. A maior atividade e densidade humana, em geral está associada a menores índices de predação (Ogada et al., 2003; Marchini et al., 2011), o que pode explicar o maior número de ataques em propriedades maiores, onde os animais podem vagar livremente a maior distância das habitações. Correlações entre ocorrências de predação e maiores plantéis e menores áreas naturais próximas às propriedades, foram observadas nas florestas tropicais do México (Amador-Alcalá et al., 2013) e em fragmentos florestais na Amazônia brasileira (Michalski et al., 2006). Em pequenas áreas naturais fragmentadas com propriedades em sua área de abrangência, o número de presas naturais reduzido e um maior número de animais domésticos fora de confinamento atraem predadores, aumentando a probabilidade de ataques (Pitman et al., 2002; Kolowski e Holekamp, 2006).
O manejo extensivo, a presença de cães e de cercas de arame foram os fatores menos significativos $(p>0,3)$. Segundo alguns autores (Pitman et al., 2002; Ogada et al., 2003; Marchini et al., 2011; Boulhosa e Azevedo, 2015), o manejo extensivo inadequado e a falta de contenção do rebanho facilitam os ataques de predadores e dificultam a observação das ocorrências de predação. Entretanto, a intensidade do manejo não mostrou correlação com os ataques (Tabela 3), possivelmente pelo sistema intensivo realizado nas propriedades servir para deter a saída de animais domésticos, porém não para impedir o avanço de animais selvagens de menor porte (Stahl et al. 2002).

Cercas de arame mostram baixa efetividade, pois, devido ao tamanho reduzido da maioria dos predadores citados, os animais conseguem passar entre os fios e/ou escalar ou escavar por baixo dos fios. A presença de cães nas propriedades não mostrou efeito repelente aos predadores, assim como encontrado por Kolowski e Holekamp (2006) na África, possivelmente por serem cães de pequeno porte e sem treinamento para guarda de rebanhos.

Assim como o indicado por 50\% (3/6) dos proprietários, a época de chuva também apareceu como período de maior ocorrência de predação em trabalhos no Quênia (Patterson et al. 2004; Kolowski e Holekamp 2006) e Guatemala (SotoShoender e Giuliano, 2011), porém mostrou correlação negativa com os ataques no Pantanal brasileiro (Michalski et al. 2006).

A dificuldade em coletar dados precisos sobre as ocorrências de predação devido à falta de registro de ataques por parte dos pecuaristas também foi observada em trabalhos no Chile (Silva-Rodriguez et al., 2009) e Brasil (Boulhosa e Azevedo, 2015). A ausência de informações precisas sobre perdas ocorre possivelmente por nenhuma das propriedades realizarem pecuária comercial, possuindo rebanhos para consumo próprio ou venda ocasional, investindo pouco em controle e registro. Sem essas informações, não é possível determinar o predador de maior importância, os fatores ambientais e zootécnicos de maior influência e nem os padrões de ocorrência (Stahl et al., 2002; Graham et al., 2004).

\section{Conclusão}

A problemática da predação em pequenas propriedades no Estado de Pernambuco apresenta diferenças significativas em comparação a outras 
regiões do Brasil e de outros países. As aves representam mais de $80 \%$ do rebanho total e estão presentes em $52 \%$ das propriedades familiares do estado e os predadores locais são animais de pequeno ou médio porte.

A instalação de cercas-vivas em propriedades próximas a áreas protegidas e o isolamento e/ou acompanhamento dos animais nas fontes de água naturais podem mostrar alguma eficácia ao menos na redução de ataques e seu baixo custo justifica sua aplicação em pequenas propriedades, servindo como pontos de partida para desenvolvimento de estratégias locais de mitigação e controle de ataques.

\section{Conflito de Interesses}

Os autores declaram não existir conflito de interesse.

\section{Agradecimentos}

À Fundação de Amparo à Ciência e Tecnologia do Estado de Pernambuco (FACEPE) pelo financiamento do "Projeto Carnívoros"; aos criadores e corpo técnico da Estação Ecológica do Tapacurá e da $10^{\mathrm{a}}$ Brigada de Infantaria Motorizada pelos dados fornecidos em entrevista e apoio nas atividades. Ao CNPq pela concessão da Bolsa de Produtividade a J. C. R. Silva.

\section{Referências}

Amador-Alcalá, S; Naranjo, E.J.; Jiménez-Ferrer, G. Wildlife predation on livestock and poultry: implications for predator conservation in the rain forest of south-east Mexico. Oryx, 47(2): 243-250, 2013.

Azevedo, F.C.C. Impact of domestic animal predation by large carnivores. How does this affect the conservation of keystone species? In: Morato, R.G.; Rodrigues, F.H.G.; Eizirik, E.; Mangini, P.R.; Azevedo, F.C.C.; MarinhoFilho, J (ed.). Manejo e conservação de carnívoros neotropicais. São Paulo: IBAMA, 2006. p.169-182.

Azevedo, F.C.C.; Murray, D.L. Evaluation of potential factors predisposing livestock to predation by jaguars. The Journal of Wildlife Management, 71(7): 2379-2386, 2007.

Boulhosa, R.L.P.; Azevedo, F.C.C. Perceptions of ranchers towards livestock predation by large felids in the Brazilian Pantanal. Wildlife Research, 41(4): 356-365, 2015.

BRASIL. IBGE - Instituto Brasileiro de Geografia e Estatística. Censo Agropecuário 2006
Agricultura familiar: Primeiros resultados, 2006. Disponível em:<http://biblioteca.ibge.gov.br/visualizacao/ periodicos/50/agro_2006_agricultura_familiar. pdf>. Acesso em: 20 de dez. 2017.

Carreira, R.S.; Petrucci-Fonseca, F. Lobo na região oeste de Trás-os-Montes (Portugal). Galemys, 12 (1): 123-134, 2000.

Distefano, E. Food and Agricultural Organization of the United Nations (FAO). Human-wildlife conflict worldwide: collection of case studies, analysis of management strategies and good practices, 2005.2 Disponível em:<http://www.fao.org/3/aau241e.pdf.>Acesso em: 16 de jan. 2018

Gese, E.M. Depredation management techniques for coyotes and wolves in North America: lessons learned and possible application to Brazilian carnivores. In: Morato, R.G.; Rodrigues, F.H.G.; Eizirik, E.; Mangini, P.R.; Azevedo, F.C.C.; Marinho-Filho, J (ed.). Manejo e conservação de carnívoros neotropicais. São Paulo: IBAMA, 2006. p. 193-214.

Graham, K.; Beckerman, A.P.; Thirgood, S. Human-predator-prey conflicts: ecological correlates, prey losses and patterns of management. Biological Conservation, 122(2): 159-171, 2004.

Hoogesteijn, R.; Hoogesteijn, A. Estratégias antipredação para fazendas de pecuária na América Latina: um guia. Campo Grande: PANTHERA, 2011, 56p.

IUCN. International Union of Conservation of Nature. The IUCN red list of threatened species, version 2018-1. Disponível em: $\langle$ http://www.iucnredlist.org $>$. Acesso em: 20 dez. 2018.

Kolowski, J.M.; Holekamp, K.E. Spatial, temporal, and physical characteristics of livestock depredations by large carnivores along a Kenyan reserve border. Biological Conservation, 128(4): 529-541, 2006.

Marchini, S.; Cavalcante, S.M.C.; De Paula, R.C. Predadores silvestres e animais domésticos: guia prático de convivência. Brasília: ICMBio, 2011. 44.p.

Mendonça, L.E.T.; Souto, C.M.; Andrelino, L.L.; Souto, W.M.S.; Vieira, W.L.S.; Alves, R.R.N. Conflitos entre pessoas e animais silvestres no Semiárido paraibano e suas implicações para conservação. Sitientibus série Ciências Biológicas, 11(2): 185-199, 2011. 
Michalski, F.; Boulhosa, R.L.P.; Faria, A.; Peres, C.A. Human-wildlife conflicts in a fragmented Amazonian forest landscape: determinants of large felid depredation on livestock. Animal Conservation, 9 (1): 179-188, 2006.

Mishra, C. Livestock depredation by large carnivores in the Indian trans-Himalaya: conflict perceptions and conservation prospects. Environmental Conservation, 24 (4): 338343, 1997.

Muñoz-Garcia, A.; Williams, J.B. Basal metabolic rate in carnivores is associated with diet after controlling for phylogeny. Physiological and Biochemical Zoology, 78 (6): 1039-1056, 2005.

Ogada, M.O.; Woodroffe, R.; Oguge, N.O.; Frank, L.G. Limiting depredation by African carnivores: the role of livestock husbandry. Conservation Biology, 17 (6): 1-10, 2003

Palmeira, F.B.L.; Barrella, W. Conflitos causados pela predação de rebanhos domésticos por grandes felinos em comunidades quilombolas na Mata Atlântica. Biota Neotropica, 7 (1): 119-128, 2007.

Patterson, B.D.; Kasiki, S.M.; Selempo, E.; Kays, R.W. Livestock predation by lions (Panthera leo) and other carnivores on ranches neighboring Tsavo National Parks, Kenya, Biological Conservation, 119(1): 507-516, 2004.
Pitman, M.R.P.L.; Oliveira, T.G.; Paula, R.C.; Indrusiak, C. Manual de identificação, prevenção e controle de predação por carnívoros. Brasília: IBAMA, 2002. 83p.

Reis, N.R.; Peracchi, A.L.; Pedro, W.A.; Lima, I.P. Mamíferos do Brasil. $2^{a}$ ed. Londrina: Edição do autor, 2006. 439p.

Silva-Rodríguez, E.A.; Soto-Gamboa, M.; OrtegaSolís, G.R.; Jiménez, J.E. Zorros, gente y gallinas: dimensión humana de un conflicto en un área rural del sur de Chile. Revista Chilena de Historia Natural, 84 (1): 375-386, 2009.

Stahl, P.; Ruette, S.; Gros, L. Predation on freeranging poultry by mammalian and avian predators: field loss estimates in a French rural area. Mammal Review, 32(3): 227-234, 2002.

Soto-Shoender, J. R.; Giuliano, W. M. Predation on livestock by large carnivores in the tropical lowlands of Guatemala. Oryx, 45(4): 561-568, 2011.

Tortato, F.R.; Layme, V.M.G.; Crawshaw Jr, P.G.; Izzo, T.J. The impact of herd composition and foraging area on livestock predation by big cats in the Pantanal of Brazil. Animal Conservation, 18(6): 539-547, 2015.

Vidolin, G.P.; Moura-Britto, M.; Braga, F.G.; Cabeças-Filho, A. Avaliação da predação a animais domésticos por felinos de grande porte no Estado do Paraná: implicações e estratégias conservacionistas. Caderno de Biodiversidade, 4 (2): 50-58, 2004. 